\title{
The Anisotropy in the Cosmic Microwave Background At Degree Angular Scales.
}

\author{
C. B. Netterfield, N. Jarosik, L. Page, D. Wilkinson, \& E. Wollack ${ }^{1}$ \\ Princeton University, Department of Physics, Jadwin Hall, P.O. Box 708, Princeton, NJ \\ 08544
}

Received —

Submitted Ap. J. Letters

${ }^{1}$ NRAO, 2015 Ivy Rd., Charlottesville, VA, 22903 


\begin{abstract}
We detect anisotropy in the cosmic microwave background (CMB) at degree angular scales and confirm a previous detection reported by Wollack et al. (1993). The root-mean-squared amplitude of the fluctuations is $44_{-7}^{+13} \mu \mathrm{K}$. This may be expressed as the square root of the angular power spectrum in a band of multipoles between $l_{e f f}=69_{-22}^{+29}$. We find $\delta T_{l}=\sqrt{l(2 l+1)<\left|a_{l}^{m}\right|^{2}>/ 4 \pi}=42_{-7}^{+12} \mu \mathrm{K}$. The measured spectral index of the fluctuations is consistent with zero, the value expected for the CMB. The spectral index corresponding to Galactic free-free emission, the most likely foreground contaminant, is rejected at approximately $3 \sigma$.

The analysis is based on three independent data sets. The first, taken in 1993, spans the 26 - $36 \mathrm{GHz}$ frequency range with three frequency bands; the second was taken with the same radiometer as the first but during an independent observing campaign in 1994; and the third, also take in 1994, spans the $36-46 \mathrm{GHz}$ range in three bands. For each telescope position and radiometer channel, the drifts in the instrument offset are $\leq 4 \mu \mathrm{K} /$ day over a period of one month. The dependence of the inferred anisotropy on the calibration and data editing is addressed.
\end{abstract}

Subject headings: cosmic microwave background - cosmology: observations 


\section{Introduction}

In Wollack et al. (1993), we reported on a measurement of the anisotropy in the CMB made from Saskatoon, SK (SK93). The results were most parsimoniously described as a detection of CMB anisotropy. However, an anisotropy in Galactic free-free emission could have produced similar data with $\approx 10 \%$ probability. We have since returned to Saskatoon and observed the same fields with an an extended frequency baseline that now spans 26 to 46 GHz. The new data, SK94, confirm the 1993 results and strongly constrain the spectral index of the fluctuations. Reviews of the state of CMB anisotropy measurements are given in Bond (1994), Readhead \& Lawrence (1992), and White et al. (1994).

\section{The Instrument and Observing Strategy}

Observations were made with two independent radiometers, one in $\mathrm{K}_{\mathrm{a}}$ band and one in $\mathrm{Q}$ band, that sequentially shared the same telescope. The details of the instrument may be found in Wollack (1994a, 1994b), Wollack et al. (1993, hereafter W1) and Page et al. (1994). Each radiometer detects the total power in three frequency bands and two polarizations? The radiation is amplified by HEMTs (Pospieszalski et al. 1988; Pospieszalski 1992 ) cooled to $15 \mathrm{~K}$. The passbands for $\mathrm{K}_{\mathrm{a}}$ band are 26-29, 29-32, 32-36 GHz, and for $\mathrm{Q}$ band are 36-39.5, 39.5-43, 43-46 GHz. The six channels in each radiometer view the sky in a single approximately frequency-independent beam formed by a cold corrugated horn feeding an ambient temperature off-axis parabola. The FWHM beam in $\mathrm{K}_{\mathrm{a}}$ band is $1.42 \pm 0.02^{\circ}$ and in $\mathrm{Q}$ band is $1.04 \pm 0.02^{\circ}$. After the parabola, the beam reflects off of a $90 \mathrm{~cm} \times 150$

\footnotetext{
${ }^{2} \mathrm{~A}$ cracked waveguide joint vitiated the data from the vertical polarization in the $\mathrm{Q}$ radiometer; it is not used.
} 
cm chopping flat that scans the beam back and forth about a vertical axis, following an approximately triangular waveform at $3.90625 \mathrm{~Hz}$. A large stationary ground screen shields the radiometer from terrestrial and solar emission. Other than the new radiometer and the modulation scheme described below, the principle improvements to the instrument described in W1 are 1) an enlarged ground screen; in our data, contaminating signals from the ground and sun are less than $1 \mu \mathrm{K} .2$ ) thermal stabilization of all radiometer components to better than $0.1 \mathrm{~K}$; and 3 ) increased vibrational isolation of the radiometers.

The chopping flat sweeps the beam in azimuth while the radiometer channels are synchronously sampled at $250 \mathrm{~Hz}$. The the angle between the sweep endpoints subtends $7.01^{\circ}$ and $7.36^{\circ}$ for the $\mathrm{K}_{\mathrm{a}}$ and $\mathrm{Q}$ systems respectively; thus, the $3 \mathrm{~dB}$ antenna beam contours of the two systems match at the extrema of their sweeps. The center of the sweep pattern is wobbled every 20 seconds from $4.42^{\circ}$ west of the north celestial pole to $4.42^{\circ}$ east.

The effective beam pattern is synthesized in software by appropriately weighting each sample during a chopper sweep. This allows us to probe multiple angular scales, optimize the spatial frequency coverage to mitigate atmospheric contamination, examine the quadrature demodulation, and match the $\mathrm{K}_{\mathrm{a}}$ and $\mathrm{Q}$ synthesized profiles even though the FWHMs are different. The full analysis based on this scheme will be discussed in a forthcoming paper; here, we use the method to synthesize the SK93 pattern. The beam pattern for one chopper sweep is shown in Figure 1. The data are normalized so that a $1 \mu \mathrm{K}$ temperature change in the positive lobe of a beam averaged over $15^{\circ}$ in right ascension ( 1 bin) gives a signal of $1 \mu \mathrm{K}$. A good approximation to the window function is $W_{l}=\left[1.5-2 P_{l}\left(\cos \left[\theta_{t}\right]\right)+.5 P_{l}\left(\cos \left[2 \theta_{t}\right]\right)\right] e^{l(l+1) \sigma^{2}}$ where $P_{l}$ are the Legendre polynomials, $\theta_{t}=2.57^{\circ}$ and $\sigma=0.64^{\circ}$.

The instrument is calibrated using Cassiopeia-A. From a fit of the 8-250 GHz data in Baars (1979) and Metzger (1986), we find $S_{\nu}=(2070 \pm 162) \nu^{(-0.695 \pm 0.029)} \mathrm{Jy},(\nu$ in GHz, 
epoch 1994) corresponding to a $9.57 \pm 1.2 \mathrm{mK}$ signal with a $1.04^{\circ}$ beam at $38.3 \mathrm{GHz}$. The laboratory calibrations agree with this to within 20\%. Because Cas-A is observed in the same thermal and radiometric environment in which the CMB is observed, we consider it the definitive calibration. Cas- $\mathrm{A}$ is also used to map the beam; the values are within $0.02^{\circ}$ of those found with a microwave source. The absolute pointing error, as determined from Cas- $\mathrm{A}$, is $0.06^{\circ}$.

\section{Data Reduction and Analysis}

In essence, the data reduction consists of editing as described below, binning the results on the sky, and subtracting the mean of the binned data. The results are shown in Figure 2. Although the constant part of the demodulated signal, or offset, depends on the east/west position and on the frequency and polarization, it is relatively independent of time. A typical drift is $3 \pm 3 \mu \mathrm{K}$ /day in both radiometers. Variations in the chopping flat temperature will effect the offset because of the dependence of the emissivity on the incident angle. The offset is fitted to the temperature of the chopper and a correlation is found; however, removing the small variation has a negligible effect on the final data set.

There are three components to the data editing procedure. 1) The data from the single-difference demodulation \&, which is sensitive to horizontal spatial gradients in the sky, are used to monitor the atmosphere. If the mean deviation of 8 s averages of data is greater than some value, $\eta$, then the 15 minute section containing those data is cut. In Table 1, we have converted $\eta$ into the standard deviation of the variation in the horizontal

\footnotetext{
3 "single-difference" refers to the demodulation where the data taken with the chopper pointed west are subtracted from those where the chopper is pointing east.
} 
thermal gradient of the atmosphere, $\zeta$. This value is relatively independent of the observing strategy. In addition, for a 15 minute section to be included, both abutting 15 minute sections must also be good. 2) Next, the standard deviation of the 64 datum from each chopper cycle is computed. If one or more points deviates by $3.5 \sigma$, then the entire chopper cycle is deleted. This cuts an additional $2.6 \%$ of the good data. 3) Finally, we require that no 20 s average of data in a right-ascension bin deviate by more than $3.5 \sigma$ of all the data in the bin. This cut rejects $\approx 1.5 \%$ of the data that passed the atmosphere cut and the final results are robust to variations in this cut level.

The data quality is monitored with a quadrature demodulation (data taken during the clockwise chopper scan minus data taken during the counterclockwise scan). This signal is consistent with zero.

To quantify the anisotropy signal, we use the Bayesian method described in W1 to find the root-mean-squared amplitude, $\Delta_{r m s}$ and index, $\beta$, of the fluctuations. In the full implementation, the new data are analyzed along with the SK93 data using a $720 \times 720$ correlation matrix. A maximum of the likelihood, $L\left(\Delta_{r m s}, \beta\right)$, is found when $\Delta_{r m s}$ and $\beta$ scale the fixed spatial correlations inherent in the observing pattern so that they best describe the patterns in the measured data. The data taken in the east and west are treated independently and the atmosphere and HEMT induced correlations are accounted for (Dodelson \& Kosowsky 1994a). The resultant $\Delta_{r m s}$ depends on the observing strategy. We convert it into an estimate of square root of the angular power spectrum in a band of multipoles between $l=47$ and $l=98\left(l_{\text {eff }}=69_{-22}^{+29}\right)$ following Bond (1994) and Peebles (1994). The results are given in Table 1. 


\section{Discussion}

Table 1 shows that there is a strong detection of anisotropy with $\beta \approx 0$, independent of atmosphere cut and data sub-set. If the $\mathrm{K}_{\mathrm{a}} 93$ data are subtracted from the $\mathrm{K}_{\mathrm{a}} 94$ data, the result is consistent with zero, as expected if both see the same signal. In other words, the $\mathrm{K}_{\mathrm{a}} 94$ results confirm the $\mathrm{K}_{\mathrm{a}} 93$ results.

There are two dominant sources of systematic error in this measurement; inaccuracy in our knowledge of the flux from Cas-A (13\%) and an inaccuracy in our measurement of Cas-A (Q: 6\%, Ka: 3\%). Small errors are introduced by inaccuracies in the beam FWHM $(2 \%)$, in the measurements of the passbands $(2 \%)$, and in the phases of the recorded signals with respect to the optical axis $(<1 \%)$. The combination of all these errors leads to a $\pm 15 \%$ uncertainty in our temperature scale. For the best estimate of celestial fluctuations we give the result with the most statistical weight $\left(44_{-6}^{+11} \mu \mathrm{K}\right)$ with the $\pm 15 \%$ added in quadrature, $\Delta_{r m s}=44_{-7}^{+13} \mu \mathrm{K}$. Converting to a band-powerf, we find $\delta T_{l}=\sqrt{l(2 l+1)<\left|a_{l}^{m}\right|^{2}>/ 4 \pi}=42_{-7}^{+12} \mu \mathrm{K}$ at $l_{\text {eff }}=69_{-22}^{+29}$.

In Table 1, one notes that the addition of new data did not improve the statistical error bar by the square root of the observing time. In part, this is because the error on $\Delta_{r m s}$ contains the sample variance, which is a function of the area of the sky observed. The statistical error bar for the $\mathrm{K}_{\mathrm{a}} 93$ data is $\approx 10.5 \mu \mathrm{K}$ (the expectation value, opposed to the maximum of the likelihood, is roughly half way between the $16 \%$ and $84 \%$ integrals). If the sample variance, which is computed to be $6.5 \mu \mathrm{K}$, is subtracted from this in quadrature then one finds an "intrinsic" error of $8.2 \mu \mathrm{K}$. The full data set should have an intrinsic error of $8.2 \sqrt{130 \mathrm{~h} / 428 \mathrm{~h} \mu} K=4.5 \mu \mathrm{K}$ which is close to the computed value of $5.5 \mu \mathrm{K}$. In

\footnotetext{
${ }^{4}$ The slight differences between Peebles (1994) and Bond (1994) may be ignored for large multipoles, $l$.
} 
addition, by inspection of Figure 2 and Table 1, one sees that the data from the three radiometers may differ by more than one would expect from random noise alone, possibly due to the atmosphere. This would tend broaden $L\left(\Delta_{r m s}, \beta\right)$.

There are two sources of a systematic error on $\beta$, uncertainty in the relative calibration between channels and the uncertainty in the fit to the Cas-A calibration data. These combine to produce an error of \pm 0.08 . In addition, we cannot be certain that atmospheric fluctuations are not biasing our answer. While the bottom two entries in Table 1 agree statistically, one might worry that low cut levels result in more negative indices. To ensure that the quoted value for $\beta$ represents one's confidence, we quote the average value of $\beta$ with an error bar that nearly encompasses the errors from both entries. Including the systematic uncertainty, $\beta=-0.1_{-0.8}^{+0.8}$.

To investigate the possibility that $\Delta_{r m s}$ is due to a Galactic foreground, we examine $L\left(\Delta_{r m s}, \beta\right)$ bearing in mind that there might be an atmospheric bias. $L\left(\Delta_{r m s}, \beta\right)$ is normalized to 1 at its maximum. For free-free emission, $\max \left[L\left(\Delta_{r m s},-2.1\right)\right]=2 \times 10^{-6}$ and $3 \times 10^{-3}$ for the bottom two entries in Table 1 respectively. For interstellar dust emission, $\max \left[L\left(\Delta_{r m s}, 1.6\right)\right]=4 \times 10^{-3}$ and $2 \times 10^{-5}$ for the same two entries. Clearly these possibilities are strongly ruled out, in the worst case at $3 \sigma$ ( $L=0.003$ for a normal distribution). In W1, $\Delta_{r m s}$ from extra-galactic sources is expected to be $\approx 9 \mu \mathrm{K}$. A conservative index for these sources is $\beta \approx-2$; thus, this explanation too is disfavored. An anisotropy in the $\mathrm{CMB}, \beta=0$, is the most compelling interpretation of the data.

In the analysis, we assume that only one celestial component produces the anisotropy. Dodelson \& Kosowsky (1994b) have performed a more general analysis on the SK93 data that allows two fluctuating components, CMB and free-free. The result was a decrease in the significance of the detection of CMB. With this new limit on $\beta, \Delta_{r m s}$ of uncorrelated 
free-free emission 9 is less than $\approx 27 \mu \mathrm{K}$, an additional constraint on two component models. Using these data alone, we cannot rule out a model which assumes co-located interstellar dust and free-free emission, but, based on FIRS (Ganga 1994) we expect dust to contribute less than $1 \mu \mathrm{K}$, again, added in quadrature.

Gaussian correlation functions are often used to compare measurements. We find $C_{0}=60_{-11}^{+14}$ for a correlation angle $\theta_{c}=1.0^{\circ}$. Alternatively, the result may be interpreted in the context of the 'standard' CDM model (Steinhardt 1993; Bond et al. 1994), $\left(\Omega_{b}=0.05, H_{0}=50 \mathrm{~km} / \mathrm{s} / \mathrm{Mpc}, \Lambda=0, n_{s}=1, \Omega=1\right.$, no reionization $)$, for which we find that the root-mean-squared quadrupole has a value $Q=18.9_{-3.6}^{+5.4} \mu \mathrm{K}$.

We would like to thank George Sofko and Mike McKibben at the The University of Saskatchewan and Larry Snodgrass at the Canadian SRC for their valuable assistance during the 1994 observing season. We are indebted to Marian Pospieszalski and Mike Ballister of NRAO who provided the amazing HEMT amplifiers that made this experiment possible. We also thank Chris Barnes, Carrie Brown, Weihsueh Chiu, Randi Cohen, Peter Csatorday, Cathy Cukras, Peter Kalmus, John Kulvicki, Wendy Lane, Young Lee, Naser Quershi, and Peter Wolanin for building many of the components of the experiment. This work was supported by NSF grant PH 89-21378, NASA grants NAGW-2801 \& NAGW-1482, and and NSF NYI grant to L. Page.

The data, analysis software, beam profile, and correlation coefficients will be made publicly available upon acceptance of this Letter.

\footnotetext{
${ }^{5}$ The free-free (or dust) contribution is estimated by assuming that it adds in quadrature to the CMB signal. We assume $\sigma_{C M B} / \sigma_{f f}=\left(\beta_{f f}-\beta\right) /\left(\beta_{C M B}-\beta\right)$. Then, $\Delta_{r m s}^{2}=\sigma_{C M B}^{2}+\sigma_{f f}^{2}$. For $\Delta_{r m s}=44 \mu \mathrm{K}$ and $\beta=-0.9, \sigma_{f f}=27 \mu \mathrm{K}$, and $\sigma_{C M B}=35 \mu \mathrm{K}$, near the bottom of our confidence range.
} 
Table 1. Summary of Results

\begin{tabular}{|c|c|c|c|c|c|c|}
\hline Data Set & $\begin{array}{l}\text { Total time } \\
\text { (h) }\end{array}$ & $\left(\mathrm{mK}{ }^{\mathrm{a}} / \mathrm{deg}\right)$ & $\begin{array}{l}\text { Mean offset } \\
\quad(\mu \mathrm{K})\end{array}$ & $\begin{array}{l}\Delta_{r m s} \mathrm{~d} \\
(\mu \mathrm{K})\end{array}$ & $\beta$ & $\begin{array}{c}\delta T_{l}^{\mathrm{e}} \\
(\mu \mathrm{K})\end{array}$ \\
\hline \multirow[t]{2}{*}{$K_{\mathrm{a}} 93^{\mathrm{f}}}$. & 130 & 4.5 & $-60 /-360$ & $37_{-9}^{+12}$ & $-0.28_{-1.06}^{+0.79}$ & $36_{-9}^{+12}$ \\
\hline & 79 & 3.0 & $\ldots$ & $44_{-10}^{+14}$ & $-0.69_{-1.16}^{+0.74}$ & $42_{-10}^{+13}$ \\
\hline $\mathrm{K}_{\mathrm{a}} 93, \mathrm{~A}-\mathrm{B}^{\mathrm{g}} \ldots \ldots$ & 130 & 4.5 & $\ldots$ & 0,31 & $\cdots$ & $\cdots$ \\
\hline \multirow[t]{2}{*}{$\mathrm{K}_{\mathrm{a}} 94$} & 140 & 2.5 & $+110 /-340$ & $49_{-7}^{+12}$ & $-0.70_{-0.74}^{+0.63}$ & $47_{-7}^{+12}$ \\
\hline & 107 & 1.7 & $\ldots$ & $52_{-8}^{+14}$ & $-0.77_{-0.83}^{+0.65}$ & $52_{-8}^{+13}$ \\
\hline 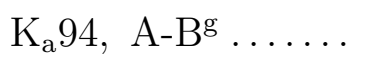 & 140 & 2.5 & $\cdots$ & 0,41 & $\cdots$ & $\cdots$ \\
\hline \multirow[t]{2}{*}{ Q94 ............. } & 158 & 2.5 & $\cdots / /-400$ & $61_{-12}^{+17}$ & $+1.35_{-2.74}^{+1.4}$ & $57_{-11}^{+16}$ \\
\hline & 98 & 1.7 & $\cdots$ & $52_{-18}^{+19}$ & $-5.97_{<-5}^{+7.23}$ & $48_{-17}^{+18}$ \\
\hline $\mathrm{K}_{\mathrm{a}} 93-\mathrm{K}_{\mathrm{a}} 94$ & 298 & $4.5,2.5$ & $\cdots$ & 0,33 & $\cdots$ & $\cdots$ \\
\hline \multirow[t]{2}{*}{$\mathrm{K}_{\mathrm{a}} 93+\mathrm{K}_{\mathrm{a}} 94$} & 298 & $4.5,2.5$ & $\cdots$ & $44_{-6}^{+11}$ & $-0.43_{-0.54}^{+0.48}$ & $42_{-6}^{+11}$ \\
\hline & 186 & $3.0,1.7$ & $\cdots$ & $49_{-7}^{+12}$ & $-0.62_{-0.59}^{+0.48}$ & $47_{-7}^{+12}$ \\
\hline $\mathrm{K}_{\mathrm{a}} 93+\mathrm{K}_{\mathrm{a}} 94, \mathrm{~A}-\mathrm{B}^{\mathrm{g}}$ & 298 & $4.5,2.5$ & $\ldots$ & 0,34 & $\cdots$ & $\ldots$ \\
\hline \multirow[t]{2}{*}{$\mathrm{K}_{\mathrm{a}} 94+\mathrm{Q} 94 \ldots \ldots$} & 298 & $2.5,2.5$ & $\ldots$ & $48_{-7}^{+11}$ & $+0.10_{-0.56}^{+0.44}$ & $44_{-6}^{+10}$ \\
\hline & 205 & $1.7,1.7$ & $\cdots$ & $51_{-8}^{+13}$ & $-0.54_{-0.69}^{+0.56}$ & $47_{-7}^{+12}$ \\
\hline \multirow[t]{2}{*}{$\mathrm{K}_{\mathrm{a}} 93+\mathrm{K}_{\mathrm{a}} 94+\mathrm{Q} 94$} & 428 & $4.5,2.5,2.5$ & $\cdots$ & $44_{-6}^{+11}$ & $+0.17_{-0.45}^{+0.42}$ & $42_{-6}^{+11}$ \\
\hline & 284 & $3.0,1.7,1.7$ & $\ldots$ & $47_{-7}^{+12}$ & $-0.40_{-0.52}^{+0.44}$ & $44_{-6}^{+11}$ \\
\hline
\end{tabular}


a Between February 21 and March 15 of 1993, the sky was observed with the $\mathrm{K}_{\mathrm{a}} 93$ radiometer for 450 hours. Between January 13, 1994 and February 28, 1994, the sky was observed for 243 hours with the $\mathrm{K}_{\mathrm{a}} 94$ radiometer data, and 363 hours with the Q94 radiometer.

${ }^{b}$ The cut level, $\zeta$ is the root-mean-squared amplitude in the horizontal temperature gradient (single-difference) for 1s of data. The larger cut levels correspond to factors of $3.1,2.5$, and 2.5 above system noise $\left(T_{\text {sys }}=T_{\text {rec }}+T_{a t m}\right)$ noise for the $\mathrm{K}_{\mathrm{a}} 93$, $\mathrm{K}_{\mathrm{a}}$ 94, and Q94 radiometers respectively. The single difference in the 1994 observing scheme is much more sensitive to atmospheric gradients than that from 1993. Data from the single-difference are not used in the anisotropy analysis. The data from the double-difference, which we analyze for anisotropy, are dominated by the system noise.

$c$ The offsets are given as (vertical polarization, A)/(horizontal polarization, B).

$d$ Data are quoted as the maximum of the likelihood with the range of the error bars encompassing the $16 \%$ to $84 \%$ integral of the likelihood marginalized with a uniform prior. Where there is no " $2 \sigma$ " lower bound, the maximum and $95 \%$ upper bound are given. The units are thermodynamic temperatures relative to a $2.726 \mathrm{~K}$ Planck emitter. At $30 \mathrm{GHz}, \partial T_{\text {ant }} / \partial T=0.98$; at $45 \mathrm{GHz}, \partial T_{\text {ant }} / \partial T=0.95$. Given our finite sampling of the CMB, presumed to be a random field, it is not possible to determine $\Delta_{r m s}$ to better than $\approx \pm 6.5 \mu \mathrm{K}$.

$e$ The synthesized beams differ by about $4 \%$ between channels. For $\mathrm{K}_{\mathrm{a}} 93$ the window spans $l_{\text {eff }}=66_{-20}^{+27}$ and $\sqrt{I(W)}=1.04$ (Bond, 1994); for $\mathrm{K}_{\mathrm{a}} 94$ the window spans $l_{\text {eff }}=67_{-20}^{+29}$ and $\sqrt{I(W)}=1.04$; and for Q94 the window spans $l_{\text {eff }}=71_{-23}^{+28}$ and $\sqrt{I(W)}=1.08 . \Delta_{r m s}=\delta T_{l} / \sqrt{I(W)}$. For the $K_{a}$ and $\mathrm{Q}$ combination, we use $l_{\text {eff }}=69_{-22}^{+29}$ and $\sqrt{I(W)}=1.06$. 
$f$ The values for the $\mathrm{K}_{\mathrm{a}} 93$ data have been recomputed given our increased understanding of Cas-A and its environs. The original values were $\Delta_{r m s}=33 \pm 10 \mu \mathrm{K}$ and $\beta=-0.3_{-1.2}^{+0.7}$. The cut level $\zeta=4.5 \mathrm{mK} / \mathrm{deg}$ corresponds to $\eta=3$ in $\mathrm{W} 1$.

$g$ This is a measure of the anisotropy of the difference between two perpendicular polarizations. It is also a gauge of the consistency between the A and B channels. The values are for $\beta=0$. 


\section{REFERENCES}

Baars, J. W. M., Genzel, R., Pauliny-Toth, I. I. K., \& Witzel, A. 1977, A\&A, 61, 99

Bond, J. R. 1994, Astrophys. Lett. \& Comm., in press. Proceedings from the Capri conference on the CMB.

Bond, J. R., et al. 1994, Phys.Rev.Lett, 72, 13

Dodelson, S. and Kosowsky, A. 1994a, FERMILAB-Pub-94/038-A, preprint

Dodelson, S. and Kosowsky, A. 1994b, Comments at the April 1994 Case Western Reserve University conference on the cosmic microwave background and FERMILAB-Pub94/308-A, preprint

Ganga, K. M. 1994, Ph.D. thesis, Princeton University

Kühr, H., Pauliny-Toth, I.I.K., Witzel, A., Schmidt, J., 1981, AJ, 86, 854-863 (S5 survey, $\left.\delta>70^{\circ}\right)$

Mezger, P. G. et al., 1986 A\&A, 167, 145

Page, L. A., et al. 1994, Proceedings from the April 1994 Case Western Reserve University conference on the cosmic microwave background

Peebles, P. J. E. 1994 ApJ, 432, L1

Pospieszalski, M. Weinreb, S., Norrod, R., \& Harris, R. 1988, IEEE, MTT, 36, 552

Pospieszalski, M. 1992, IEEE, MTT-S, 1369

Readhead, A. C. S., and Lawrence, C. R. 1992, ARA\&A, 30, 653

Steinhardt, P. J. 1993, Private Communication 
White, M., Scott, D., and Silk, J. 1994, ARA\&A, 32, 319

Wollack, E. J., et al. 1993, ApJ, 419, L49

Wollack, E. J., 1994a. Astrophys. Lett. \& Comm., proceedings from the Capri conference on the CMB.

Wollack, E. J. 1994b, Ph.D. thesis, Princeton University

This manuscript was prepared with the AAS LATEX macros v3.0. 


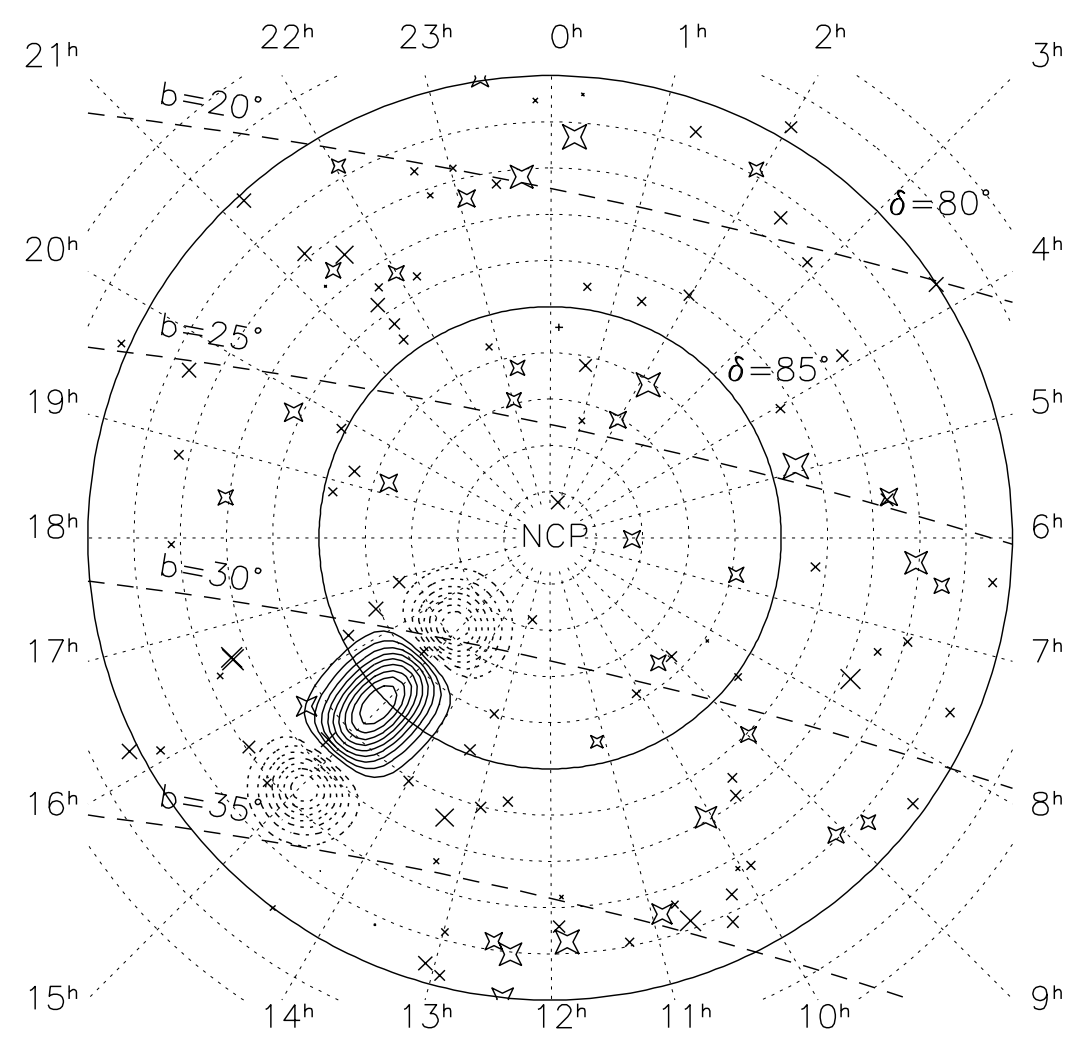

Fig. 1. - The region of observation and beam pattern for SK93. The contours show the beam profile for 1 s of data. In the analysis, all the data with the beam center within one hour of right ascension are combined; thus, the above beam pattern is smeared out. Note how much the pattern resembles a "double-difference" (positive lobe:solid lines; negative lobes: dashed lines). The '+' signs mark the sources in Kühr et al. (1981). Stars mark the sources with flat spectra. The size of the symbol is proportional to the flux. Lines of constant galactic latitude are also shown. Note the slight curvature of the observing pattern with respect to lines of constant longitude; this is a result of the azimuthal chop. Note that if there were a hot spot in the CMB near $\delta=87^{\circ}$ and $\alpha=5^{h}$, the data in bins 4 through 8 would tend to be low. 


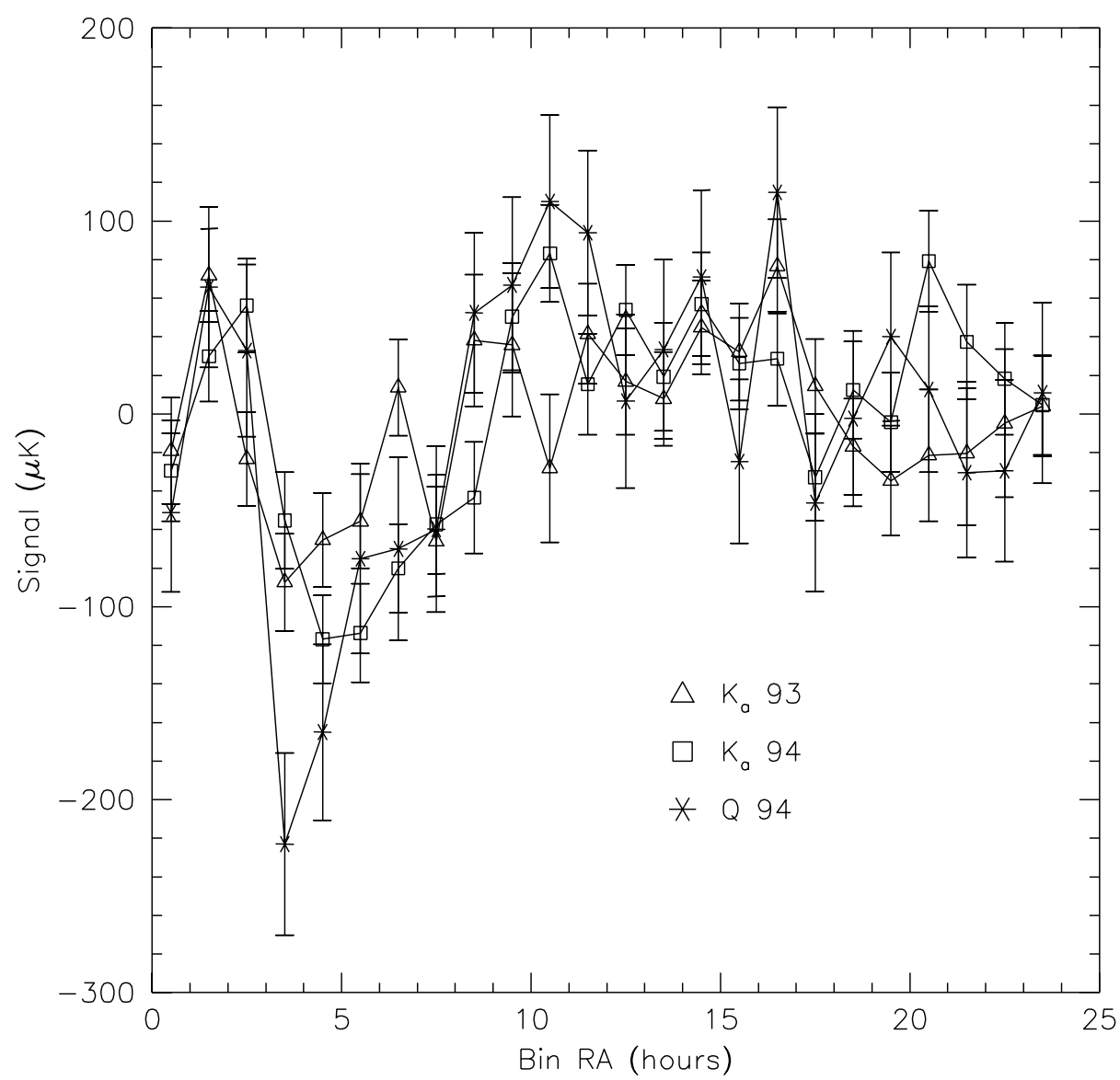

Fig. 2. - The data for $\mathrm{K}_{\mathrm{a}} 93, \mathrm{~K}_{\mathrm{a}} 94$, and $\mathrm{Q} 94$ as a function of right ascension bin. Data from the six $\mathrm{K}_{\mathrm{a}}$ bands have been added together as have the data from the three $\mathrm{Q}$ bands. Also, the data taken in the east have been combined with the data taken in the west. The error bars include the effects of the correlations between channels. There are no correlations, other than those from the CMB, between the three data sets. Because of the observing strategy, each bin is correlated with the adjacent bins. All correlations have been accounted for in the analysis. 